\title{
Recuperación energética y análisis térmico del proceso de cocción en la industria cerámica
}

\section{Energetic recovery and thermal analysis of the cooking process in the ceramic industry}

\author{
Ana Medina-Jiménez ${ }^{1 a}$, Julián Jaramillo ${ }^{1 b}$ \\ ${ }^{1}$ Universidad Industrial de Santander, Colombia. Orcid: a0000-0002-8024-2880, b0000-0001-7389-2677 \\ Correos electrónicos: acaromedina88@ hotmail.com, bjejarami@uis.edu.co \\ Recibido: 11 septiembre, 2016. Aceptado: 5 marzo, 2018. Versión final: 21 junio, 2018.
}

\begin{abstract}
Resumen
Las investigaciones encontradas en la literatura no son específicas para hornos colmena, que son los más usados en la industria ladrillera (cerámica) en Colombia. Es por esto que en este trabajo se desarrolla un modelo matemático analítico que simula el comportamiento de los gases de combustión en esos hornos. En el horno se modela la combustión, la transferencia de calor y masa a los productos y las pérdidas por las paredes. El modelo matemático usado es validado con resultados experimentales. Además, con los datos experimentales medidos en el ducto de descarga y chimenea, se selecciona un sistema recuperador para aprovechar la energía residual.
\end{abstract}

Palabras clave: combustión de carbón, horno colmena, intercambiador de calor termodinámica, transferencia de calor.

\begin{abstract}
Previous scientific research has not focused on downdraught beehive kilns, which are the most used in the Colombian brick (ceramic) industry. Hence, in the present paper, an analytical mathematical model is developed in order to simulate the exhaust gas behavior. To do so, gas combustion and heat and mass transfer to the products and the walls are modelled within the kiln. The obtained model is validated by experimental data. Finally, with the experimental data acquired in the discharge duct and chimney, a heat recovery system is proposed to make use of the residual energy.
\end{abstract}

Keywords: heat transfer, mathematical model, downdraught beehive kiln, coal combustion, thermodynamics.

\section{Introducción}

La industria cerámica colombiana, considerada como una industria tradicional por el gremio, no ha tenido grandes avances tecnológicos en los últimos años. Por este motivo es necesario desarrollar estudios con miras a mejorar el proceso y aumentar la competitividad y la eficiencia [1,2]. El proceso de fabricación de un producto cerámico implica varias etapas sucesivas: 1) preparación de la materia prima; 2) moldeo de las piezas; 3 ) secado; 4) caldeo (cocción previa) y 5) quema plena (cocción completa) [3].
Los hornos son empleados para la cuarta y la quinta etapa. En la cuarta etapa de cocción, llamada caldeo, se retira la humedad de los productos y se eleva la temperatura del horno hasta $200{ }^{\circ} \mathrm{C}$. Esta etapa dura un poco más de un día ( 25 horas). En la quema plena o etapa final de cocción, la temperatura dentro del horno alcanza $900{ }^{\circ} \mathrm{C}$. En esta, la pieza cerámica adquiere propiedades como dureza, estabilidad, resistencia mecánica y química, entre otras.

ISSN impreso: 1657 - 4583, ISSN en línea: 2145 - 8456, CC BY-ND 4.0 (c) () $९$

Como citar: A. Medina-Jiménez, J. Jaramillo, "Recuperación energética y análisis térmico del proceso de cocción en la industria cerámica," Rev. UIS Ing., vol. 18, no. 1, pp. 81-98, 2019.doi: 10.18273/revuin.v18n1-2019007 


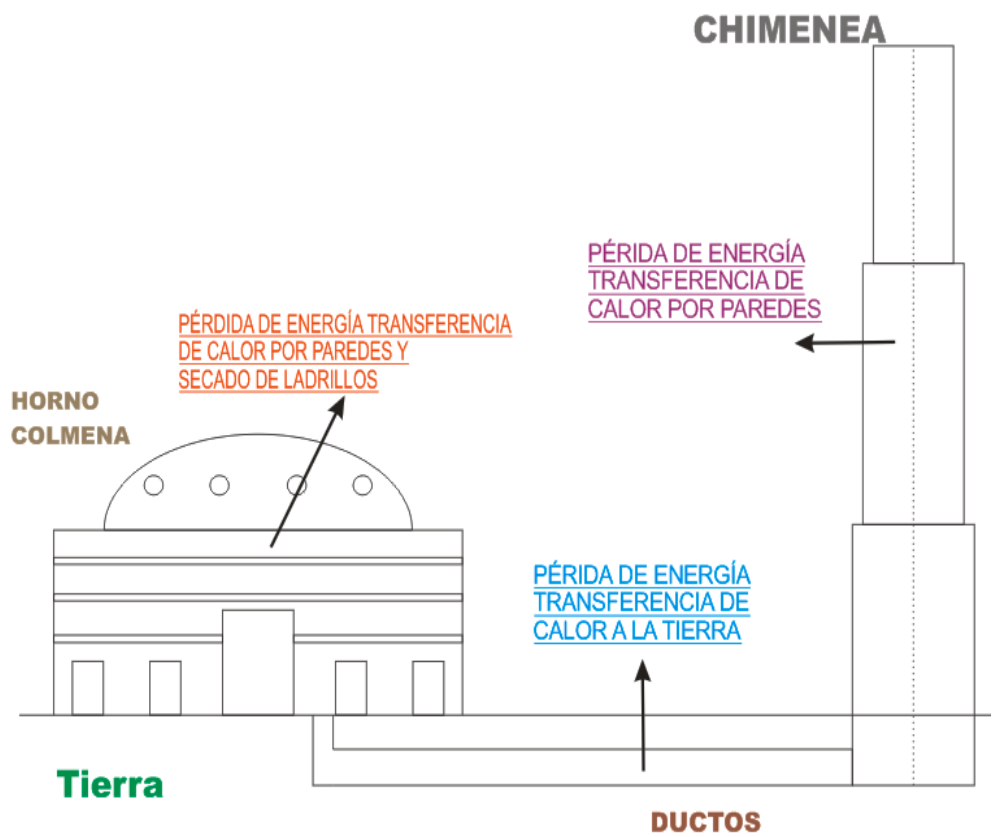

Figura 1. Elementos de la planta analizada y representación de las pérdidas de energía.

La duración aproximada es en promedio de dos días (42 horas). Existen varios tipos de hornos comúnmente usados para la fabricación de productos cerámicos, sin embargo, el más tradicional en Colombia es el horno colmena u horno brasilero. Son del tipo fijo, de calcinación interna y de producción en tandas. Son circulares, con techo en cúpula o domo, y están construidos con ladrillos comunes. La pared circular está totalmente en contacto con el aire exterior. Como se observa en la figura 1, el horno tiene una serie de ductos que llevan los gases desde su base hasta la chimenea, que, por tiro natural, se encarga de extraerlos del sistema y llevarlos al ambiente [4]. En cada uno de estos sistemas se tienen pérdidas de calor.

Algunos autores han desarrollado diversos trabajos para intentar representar los principales fenómenos que se presentan en los hornos. En el trabajo realizado por [5], se proporciona un estudio detallado de los hornos para la cocción de productos cerámicos, y se presentan los elementos más importantes para el modelado, el diseño y los procesos de transferencia de calor involucrados. Las pérdidas de energía por las paredes del horno se deben a que los gases intercambian calor con ellas por convección natural y radiación, de modo que la temperatura de los gases disminuye. En los trabajos presentados por [6,7], se suministran unos estudios de los procesos involucrados en la combustión, y se presentan de forma detallada los componentes que hacen parte de la combustión del carbón, que es el combustible utilizado en este proceso.
Algunos autores presentan correlaciones que se pueden aplicar para modelar estos fenómenos de transferencia de calor en el análisis del horno [8,9]. El intercambio de calor entre los gases y los productos se debe también a la transferencia de calor por convección y radiación. La energía ganada por los productos se utiliza para evaporar el agua presente en ellos, lo que constituye un fenómeno de transferencia de masa [9]. Otro aspecto a considerar en el horno es la caída de presión, que debe ser compensada por el diferencial de presión generado por el tiro natural que ejerce la chimenea para extraer y enviar los gases a la atmósfera $[10,11]$.

Entender los procesos físicos y térmicos presentes en el horno durante la etapa de cocción en la producción de piezas cerámicas permite determinar los lugares donde hay oportunidades de ahorro y donde sería productivo implementar algún dispositivo para recuperar energía. En la industria existen diferentes sistemas recuperadores de calor, que permiten aprovechar este calor residual. Un artículo reciente [12] ilustra la instalación de un intercambiador de calor (utilizando aceite térmico como fluido de intercambio) en la chimenea de un horno continuo de gas en una ladrillera, con el objetivo de mejorar el aprovechamiento de la energía consumida durante la cocción y aprovecharla en el proceso de secado. En la industria colombiana, este proceso se realiza a una temperatura de entre $60{ }^{\circ} \mathrm{C}$ y $90{ }^{\circ} \mathrm{C}$. Si el proceso se hace a menos de $60{ }^{\circ} \mathrm{C}$, no se observaría la utilidad de extraer el calor de los gases, y si se hace a más de $90{ }^{\circ} \mathrm{C}$, se pueden generar fisuras en los productos [13]. 


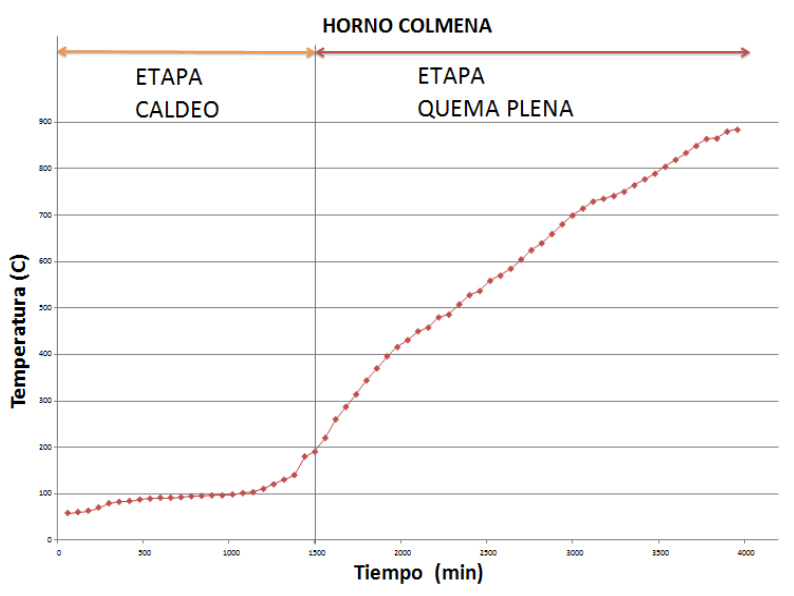

Figura 2. Etapas de cocción horno colmena

El presente trabajo tiene dos objetivos. El primero es desarrollar un modelo matemático capaz de simular el comportamiento de los gases dentro del horno colmena durante la primera y la segunda etapa de cocción, ya que estas etapas del proceso son las de mayor consumo energético entre las utilizadas para la fabricación de productos cerámicos. Por tanto, el horno es el elemento donde más se presenta consumo de energía, puesto que dentro de él se deben alcanzar temperaturas de hasta $1.000{ }^{\circ} \mathrm{C}$, para cumplir con los requerimientos del proceso (figura 2). Entonces, es importante tener claro cuáles son los fenómenos térmicos presentes en el horno colmena y su funcionamiento. El segundo es diseñar un recuperador de calor para aprovechar la energía residual en la etapa de quema plena.

Se realizó un estudio experimental en un horno colmena de una empresa ladrillera representativa. Los datos obtenidos se usaron para validar el modelo matemático desarrollado. Con base en los resultados obtenidos, se presentaron cinco posibles alternativas para el sistema recuperador de calor, y se seleccionó la mejor alternativa. Se realizaron mediciones de temperatura en diferentes puntos del proceso, con los siguientes propósitos: i) verificar que las temperaturas sean adecuadas para la implementación del sistema recuperador y poder usarlas en el diseño, ii) para validar los resultados obtenidos en el modelo matemático desarrollado.

\section{Materiales y métodos}

\subsection{Modelo matemático}

En esta sección se presentan las ecuaciones, las variables, los parámetros utilizados y el modelo matemático desarrollado para simular el proceso de transferencia de calor y masa en el horno. Se asumirá un consumo de
176.32 (kg-carbón/kg-arcilla-cocida), para producir 127 toneladas de producto (ladrillos y tejas) [4]. Además, para el desarrollo del modelo se asume que:

- El flujo es estacionario.

- Las propiedades físicas de los gases de combustión varían con la temperatura.

- La transferencia de calor entre el gas y los productos y el gas y las paredes es por convección y radiación.

- La temperatura tanto de los productos como de las paredes y de los gases es uniforme dentro del horno.

- El suministro de carbón es función del tiempo.

- La acumulación de agua presente en los productos no se tiene en cuenta.

- Se desprecian las variaciones por energía cinética y potencial.

- Medio participante en la radiación, utilizando los modelos de radiación de un gas gris isotérmico en un recinto formado por dos superficies grises y radiación entre un gas y un recinto de una sola superficie gris[14].

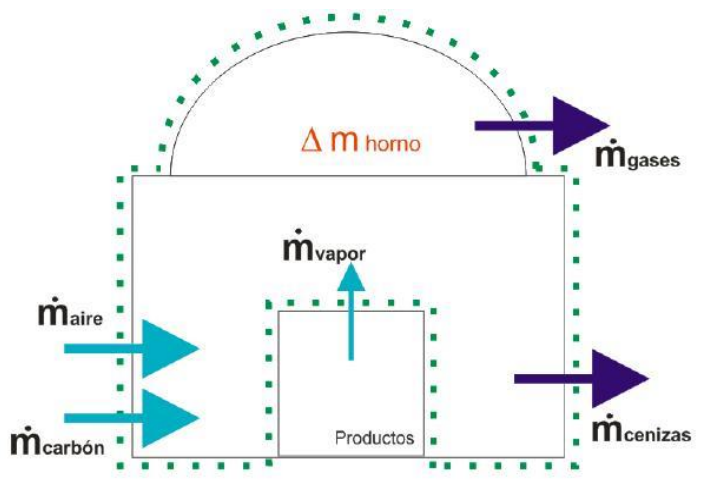

Figura 3. Flujos másicos dentro del horno

Para caracterizar los fenómenos presentes en el horno, es necesario utilizar como volumen de control de este mismo horno. En este volumen de control es posible identificar de forma clara los aportes energéticos del aire 
y el combustible, las pérdidas de calor por paredes, las cenizas, la transferencia de calor y masa entre gases y productos y la acumulación de calor dentro del horno (figura 3). Se realiza un balance de masa, ecuación (1), con el fin de identificar los flujos másicos de las distintas corrientes de entrada y salida del sistema. Así como, las cantidades totales y las composiciones medias que están en el mismo, en un instante dado.

$$
\frac{d(\rho V)_{v c}}{d t}=m_{\text {aire }}+m_{\text {carbón }}+m_{\text {vapor }}+m_{\text {cenizas }}+m_{\text {gases }}
$$

Donde

$\dot{\boldsymbol{m}}_{\text {aire }}$ es el flujo másico del aire de combustión $\left[\mathrm{kg} \mathrm{s}^{-1}\right]$.

$\dot{\boldsymbol{m}}_{\boldsymbol{c a r b o ́ n}}$ es el flujo másico del carbón usado en el proceso $\left[\mathrm{kg} \mathrm{s}^{-1}\right]$.

$\dot{\boldsymbol{m}}_{\text {vapor }}$ es el flujo másico del vapor extraído de los productos durante el proceso de cocción $\left[\mathrm{kg} \mathrm{s}^{-1}\right]$.

$\dot{\boldsymbol{m}}_{\text {cenizas }}$ es el flujo másico de las cenizas producto de la combustión $\left[\mathrm{kg} \mathrm{s}^{-1}\right]$.

$\dot{\boldsymbol{m}}_{\text {gases }}$ es el flujo másico de gases generados en la combustión $\left[\mathrm{kg} \mathrm{s}^{-1}\right]$.

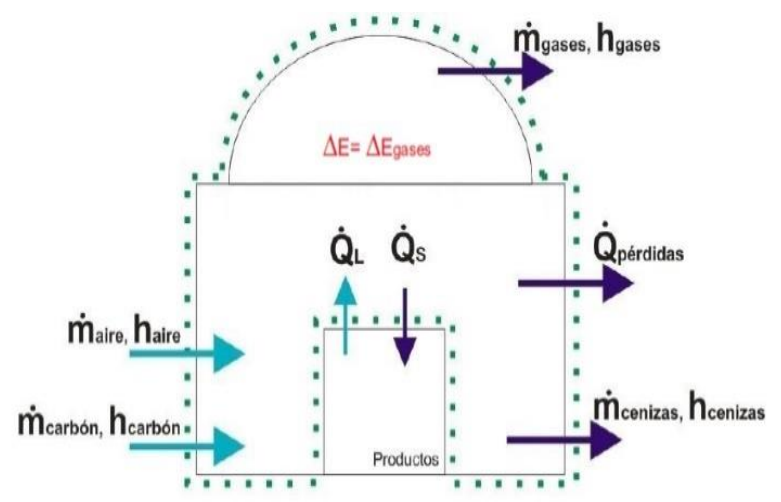

Figura 4. Flujos de energía dentro del horno.

El balance de energía permite determinar la cantidad de energía que se intercambia y se acumula dentro del sistema. Además, es posible obtener una aproximación del valor de la temperatura en el horno, a lo largo del paso del tiempo. En este trabajo, se tiene en cuenta la energía entregada por la combustión del carbón, el calor latente y sensible intercambiado entre los productos y los gases de combustión dentro del horno, las pérdidas de calor por paredes, la energía pérdida en las cenizas del carbón y la energía que los gases conservan, como se muestra en la figura 4. Además, para obtener la composición de los gases producidos en la combustión, se realiza un análisis estequiométrico. Para lo cual, se tiene en cuenta el carbón suministrado, el análisis último de este y el exceso de aire de $120 \%$. Con estos datos se hace el balance de la ecuación de la combustión. Así se obtiene el aporte de cada gas para el cálculo de las propiedades termofísicas.
En la tabla 1 se presenta el análisis último en base seca (b. s.) del carbón utilizado en el análisis, así como otras características importantes.

Tabla 1. Características del carbón

\begin{tabular}{|c|c|}
\hline Componente & \% masa (b.s.) \\
\hline Carbono (C) & 60,3 \\
\hline Hidrogeno $(\mathrm{H})$ & 4,9 \\
\hline Nitrógeno $(\mathrm{N})$ & 1,0 \\
\hline Oxígeno $(\mathrm{O})$ & 13 \\
\hline Cenizas $(\mathrm{Cz})$ & 19,6 \\
\hline Azufre (S) & 1,2 \\
\hline Humedad & 10 \\
\hline Poder calorífico inferior $\left(q_{c i}\right)$ & $29,5\left[\mathrm{~kJ} \mathrm{~kg}^{-1}\right]$ \\
\hline
\end{tabular}

El balance de energía, para la mezcla aire-gases dentro del horno, puede escribirse así:

$$
\begin{aligned}
& \frac{d\left(E_{v c}\right)}{d t}=(m h)_{\text {aire }}+m_{\text {carbór }} q_{c i}+Q_{L}-Q_{S} \\
& -(m h)_{\text {cenizas }}-(m h)_{\text {gases }}-Q_{\text {pérdidas }}
\end{aligned}
$$

Donde

- $h_{\text {aire }}$ es la entalpía del aire de combustión $\left[\mathrm{kJ} \mathrm{kg}^{-1}\right]$.

- $q_{c i}$ es el poder calorífico inferior del carbón $\left[\mathrm{kJ} \mathrm{kg}^{-1}\right]$.

- $\dot{Q}_{L}$ es el calor producto del vapor extraído de los productos durante el proceso de cocción $\left[\mathrm{kJ} \mathrm{kg}^{-1}\right]$.

- $\dot{Q}_{S}$ es el calor transferido a los productos $\left[\mathrm{kJ} \mathrm{kg}^{-1}\right]$.

- $h_{\text {cenizas }}$ es la entalpía de las cenizas producto de la combustión [ $\left.\mathrm{kJ} \mathrm{kg}^{-1}\right]$.

- $h_{\text {gases }}$ es la entalpía de los gases producto de la combustión $\left[\mathrm{kJ} \mathrm{kg}^{-1}\right]$.

La acumulación de energía dentro del horno se modela de acuerdo con la ecuación (3):

$$
\frac{d\left(E_{v c}\right)}{d t}=m_{\text {gases }}\left(C_{v, g a s}\left(T_{g}^{i+1}\right)-C_{v, g a s}\left(T_{g}^{i}\right)\right)
$$

Donde $C_{v_{\text {gas }}}\left(T_{g}^{i+1}\right)\left[\mathrm{kJ} \mathrm{kg}^{-1}\right.$ ] es el calor específico a volumen constante de los gases a la temperatura de los gases en el instante de tiempo $(i+1)$ y $C_{v_{\text {gas }}}\left(T_{g}^{i}\right)$ en el instante de tiempo $i$. Las pérdidas de calor asociadas a las paredes del horno se obtienen realizando el balance de energía en la superficie de ellas. Los gases sufren una pérdida de energía por las paredes del horno, el calor es cedido a las paredes por convección y radiación (figura 5). Además, en las paredes del horno se tiene en cuenta la acumulación y la conducción. La ecuación (4) representa las pérdidas consideradas. 


$$
Q_{\text {pérdidas }}=Q_{\text {pérdidaspared }}=Q_{\text {conv }, w-\text { gas }}+Q_{\text {rad,w-gas }}
$$

Para predecir las temperaturas de las paredes interior y exterior del horno, se utiliza el método de diferencias finitas, como solución numérica para la conducción transitoria en las paredes del horno. Se asume que la pared está dividida en secciones $\Delta \mathrm{x}$, de modo que resultan dos nodos para analizar (figura 5). Se aplica el balance de energía en régimen transitorio, ecuación (5), para el elemento de volumen del nodo superficial interior $\mathrm{N}_{\text {int }} \mathrm{y}$ para el nodo exterior $\mathrm{N}_{\mathrm{ext}}$.

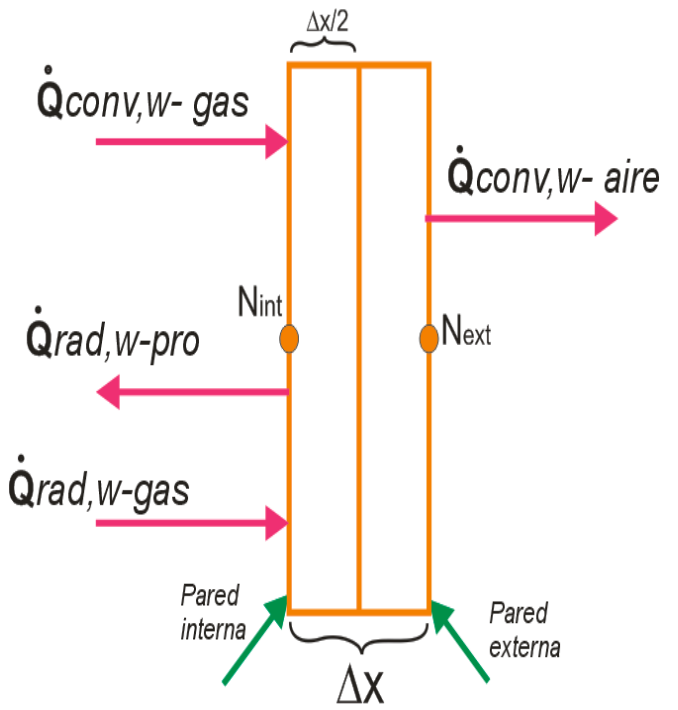

Figura 5. Flujos de calor en las paredes

$$
\begin{aligned}
& Q_{\text {conv }, w-g a s}+Q_{\text {rad,w-gas }}-Q_{\text {rad,w-pro }}-Q_{\text {cond }, \text { Nint-Next }} \\
& =\rho_{w} A_{w}\left(\frac{\Delta x}{2}\right) C_{p, w}\left(\frac{T_{w i}^{i+1}-T_{w i}^{i}}{\Delta t}\right)
\end{aligned}
$$

\subsubsection{Calor por convección entre los gases y la pared interior del horno}

$$
Q_{c o n v, w-g a s}=h_{c w} A_{w}\left(T_{g}^{i}+T_{w i}^{i}\right)
$$

$h_{c w}$ es el coeficiente de convección entre los gases y la pared $\left[\mathrm{W} / \mathrm{m}^{2} \mathrm{~K}\right]$, y se calcula con la ecuación (7).

$$
h_{c w}=\frac{N_{u} K_{g}^{i}}{L_{c}} \quad \text { Donde }
$$

- $K_{g}^{i}$ es la conductividad térmica de los gases en el horno [W/m K].

- $L_{c}$ es la longitud característica del horno en el interior [m].
$N_{u}$ es el número de Nusselt [16], y se calcula con la ecuación (8).

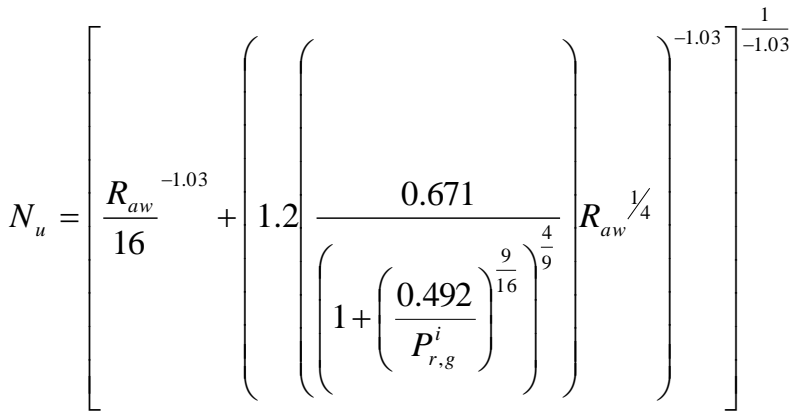

$R_{a w}$ es el número de Rayleigh para el interior del horno. Y se calcula usando la ecuación (9).

$$
R_{a w}=\left(\frac{g_{r}\left(\frac{2}{T_{g}^{i}+T_{w}^{i}}\right)\left(T_{g}^{i}-T_{w}^{i}\right) L_{c}}{\left(\frac{\mu_{g}^{i}}{\rho_{g}^{i}}\right)^{2}}\right) P_{r, g}^{i}
$$

$P_{r, g}^{i}$ es el número de Prantl de los gases en el horno, y se calcula con la ecuación (10).

$$
P_{r, g}^{i}=\frac{\mu_{g}^{i} C_{p, g}\left(T_{g}^{i}\right)}{K_{g}^{i}}
$$

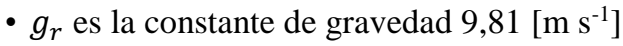

- $\mu_{g}^{i}$ viscosidad dinámica de los gases $[\mathrm{kg} / \mathrm{m} \mathrm{s}]$

- $\rho_{g}^{i}$ densidad de los gases $\left[\mathrm{kg} / \mathrm{m}^{3}\right]$

- $T_{g}^{i}$ es la temperatura del gas en el interior del horno en el primer instante $\left[{ }^{\circ} \mathrm{C}\right]$.

- $T_{w i}^{i}$ es la temperatura de la pared interior del horno en el primer instante $\left[{ }^{\circ} \mathrm{C}\right]$.

- $T_{w i}^{i+1}$ es la temperatura de la pared interior del horno a en el instante de tiempo $(i+1)\left[{ }^{\circ} \mathrm{C}\right]$.

- $A_{w}$ área superficial interna del horno colmena $\left[\mathrm{m}^{2}\right]$.

- $\rho_{w}$ densidad del material de las paredes $\left[\mathrm{kg} / \mathrm{m}^{3}\right]$

- $C_{p}, w$ poder calorífico del material de las paredes [kJ $\left.\mathrm{kg}^{-1}\right]$

- $C_{p, g}\left(T_{g}^{i}\right)$ es el poder calorífico de los gases a la temperatura de los gases en el instante de tiempo i $\left[{ }^{\circ} \mathrm{C}\right]$. 


\subsubsection{Radiación entre los gases y la pared interior del horno [14]}

$$
Q_{r a d, w-g a s}=\frac{A_{w} \sigma \varepsilon_{w}\left(\varepsilon_{g}^{i} T_{g}^{i^{4}}-\alpha_{g}^{i} T_{w i}^{i^{4}}\right)}{\left(\varepsilon_{w}+\alpha_{g}^{i}\right)-\left(\varepsilon_{w} \alpha_{g}^{i}\right)}
$$

Donde

- $\sigma$ es la constante de Stefan-Boltzmann $5.67 \times 10^{-8}$ $\left[\mathrm{W} / \mathrm{m}^{2} \mathrm{~K}^{4}\right]$.

- $\varepsilon_{w}$ es la emisividad de las paredes.

- $\varepsilon_{g}^{i}$ es la emisividad de los gases en el horno a la temperatura de los gases en el instante de tiempo i.

- $\alpha_{g}^{i}$ es la absortividad de los gases a la temperatura de los gases en el instante de tiempo i.

\subsubsection{Por radiación entre la pared y los productos en el interior del horno}

Donde

$$
Q_{r a d, w-p r o}=\frac{\sigma\left(T_{w i}^{i^{4}}-T_{p r}^{i^{4}}\right)}{R_{t}}
$$

- $T_{p r}^{i}$ es la temperatura de los productos en el instante de tiempo i $\left[{ }^{\circ} \mathrm{C}\right]$.

- $R_{t}$ es la resistencia térmica equivalente para la radiación, que se calcula con la ecuación (13) [14].

- $A_{w}$ es el área de la pared interna del horno $\left[\mathrm{m}^{2}\right]$.

- $A_{p r}$ es el área de los productos dentro del horno $\left[\mathrm{m}^{2}\right]$.

$$
R_{t}=\frac{\frac{1}{\left(1-\varepsilon_{g}^{i}\right) A_{w}}\left(\frac{1}{\varepsilon_{g}^{i} A_{w}}+\frac{1}{\varepsilon_{g}^{i} A_{p r}}\right)}{\frac{1}{\left(1-\varepsilon_{g}^{i}\right) A_{w}}+\frac{1}{\varepsilon_{g}^{i} A_{w}}+\frac{1}{\varepsilon_{g}^{i} A_{p r}}}
$$

2.1.4. Calor por convección natural entre el aire ambiente y la pared exterior del horno

$$
Q_{\text {conv }, w-a i r e}=h_{c, w e x} A_{\text {wex }}\left(T_{\text {wex }}^{i}-T_{a}\right)
$$

Donde

- $h_{c, \text { wex }}$ es el coeficiente de convección entre el aire ambiente y la pared exterior $\left[\mathrm{W} / \mathrm{m}^{2} \mathrm{~K}\right]$. Se calcula usando las ecuaciones (7) a (10).

- $A_{\text {wex }}$ es el área superficial externa del horno colmena $\left[\mathrm{m}^{2}\right]$.

- $T_{\text {wex }}^{i}$ es la temperatura de la pared exterior del horno $\left[{ }^{\circ} \mathrm{C}\right]$.

- $T_{a}$ es la temperatura del aire ambiente $\left[{ }^{\circ} \mathrm{C}\right]$.

\subsubsection{Calor por conducción a través de la pared del} horno

$$
Q_{\text {cond }, N_{\mathrm{int}}-N_{\text {ext }}}=k_{w} S\left(T_{w i}^{i}-T_{w e x t}^{i}\right)
$$

Donde

- $k_{w}$ es la conductividad térmica de la pared del horno [W/m K].

- $S$ es el factor de forma de conducción en régimen estacionario, evaluado con la ecuación [14].

- $D_{2}$ es el diámetro externo del horno [m].

- $D_{1}$ es el diámetro interno del horno [m].

$$
S=\frac{2 \pi L_{c}}{\ln \left(\frac{D_{2}}{D_{1}}\right)}
$$

La energía entregada a los productos se debe también a la transferencia de calor por convección y radiación de los gases (figura 6). Esta energía, ganada por los productos, se utiliza para evaporar el agua presente en los productos, lo que constituye un fenómeno de transferencia de masa.

Con este balance se obtiene una aproximación del valor de la temperatura en la superficie de los productos en el tiempo (ecuación (17). Se puede observar que como la temperatura de los gases es mayor que la de los productos, se produce una transferencia de calor sensible hacia los productos, y que la humedad que está siendo removida de los productos hacia los gases, se trasforma en un flujo de calor latente. Estos fenómenos se representan en la ecuación

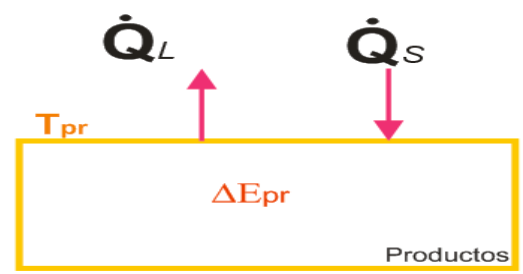

Figura 6. Flujos de calor en productos

$$
\begin{aligned}
& \left(m_{p} C_{p p}\right) \frac{d T}{d t}=\left(m_{p} C_{p p}\right) \frac{\left(T_{p r}^{i+1}-T_{p r}^{i}\right)}{\Delta t}=Q_{s}-Q_{L} \\
& Q_{s}=Q_{\text {conv }, \text { gas }-p r}+Q_{\text {rad,gas }-p r}+Q_{\text {rad }, w-p r}
\end{aligned}
$$

2.1.6. Calor por convección entre los gases y los productos

$$
Q_{\text {conv,gas- } p r}=h_{c g} A_{p r}\left(T_{g}^{i}-T_{p r}^{i}\right)
$$




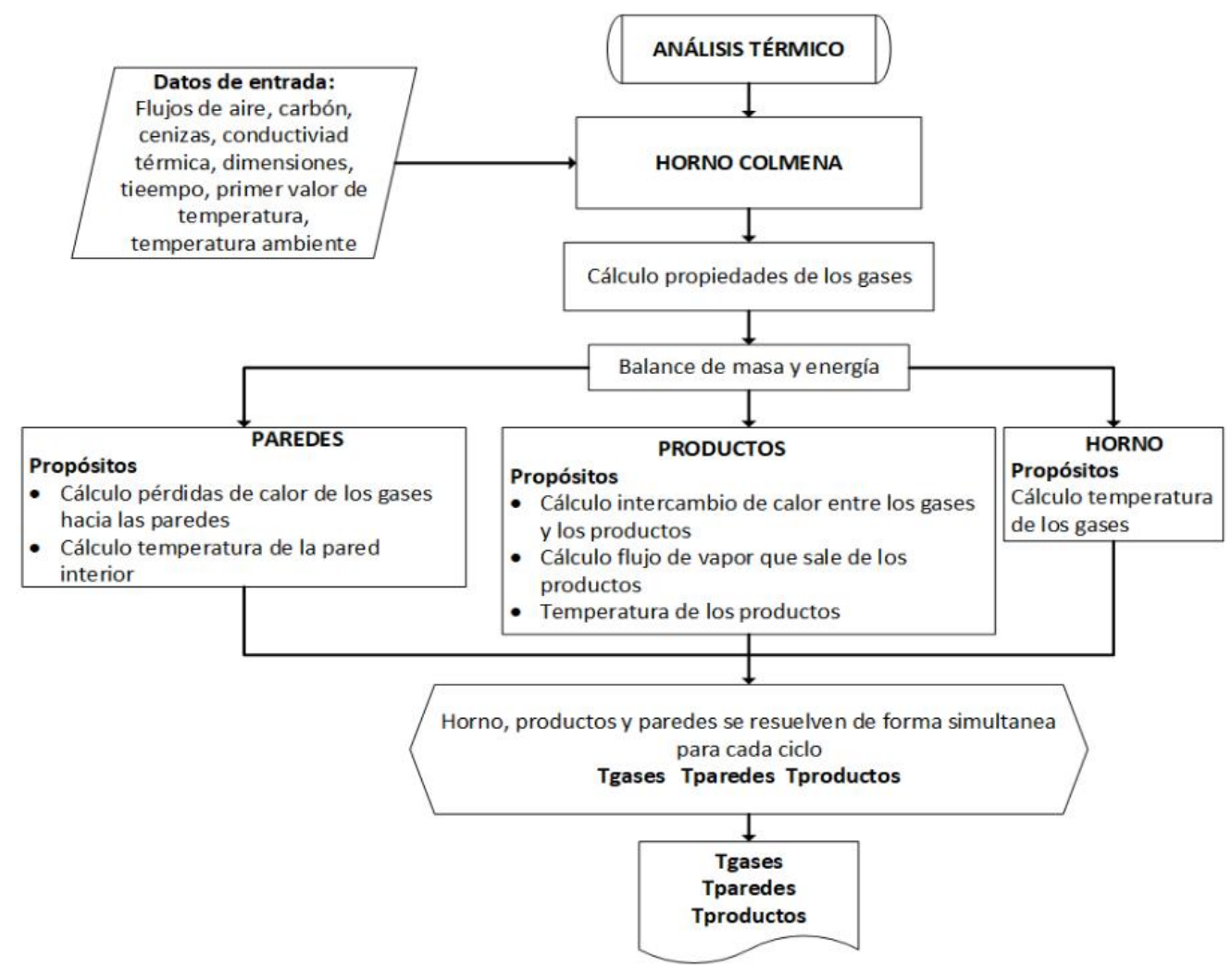

Figura 7. Algoritmo global de solución

$h_{c_{g}}$ es el coeficiente de transferencia de calor convectivo [W/ $\left./ \mathrm{m}^{2} \mathrm{~K}\right]$, se evalúa utilizando las ecuaciones (7) a (10).

El calor intercambiado por radiación entre los gases y los productos $\left(\dot{Q}_{\text {rad,gas-pr }}\right)$ se calcula aplicando los valores de la temperatura de los productos en la ecuación (11).

\subsubsection{Calor latente que sale de los productos}

$$
Q_{L}=m_{v} h_{f g}
$$

Donde

- $\dot{m}_{v}$ masa de vapor que sale de los productos en el tiempo $\left[\mathrm{m} \mathrm{s}^{-1}\right]$.

- $h_{f g}$ entalpía de evaporación del agua a la temperatura de los productos $T_{p r}^{i}\left[\mathrm{~kJ} \mathrm{~kg}^{-1}\right]$.

No se considera el sobrecalentamiento que sufre el vapor al elevar su temperatura por encima de la temperatura de saturación. En la tabla 2 se relacionan los principales parámetros usados en el modelo matemático. Los datos de sistema horno-ductos-chimenea fueron tomados de una empresa ladrillera de la región que usa horno colmena. En esta empresa se realizaron los estudios experimentales.
Todos los balances y las ecuaciones se resuelven de forma secuencial para el horno. Las propiedades físicas de los gases varían con la temperatura. El modelo matemático fue desarrollado en el programa Scilab [17]. En la tabla 2 se muestra el algoritmo global de solución.

\subsection{Estudios experimentales}

Se realizaron mediciones de temperatura en diferentes puntos del proceso para determinar las temperaturas alcanzadas en la etapa de cocción. Para esto se instalaron termopares en diferentes puntos del proceso, que están representados en la figura 8: (T1) temperatura dentro del horno a una altura de $2 \mathrm{~m}$ y a $0,5 \mathrm{~m}$ de la pared interior aproximadamente, (T2) temperatura en el punto medio del ducto a la salida del horno, (T3) temperatura en el punto medio en la base de la chimenea, (T4) temperatura a una altura de 7,5 m medidos desde la base de la chimenea. Estos datos se usan con un doble propósito: i) verificar que las temperaturas sean adecuadas para la implementación del sistema recuperador y usarlas en su diseño, ii) para compararlas con los resultados obtenidos del modelo matemático desarrollado. Para las pruebas, se instalaron los equipos en la fábrica y se realizaron mediciones para el proceso completo de quema (caldeo, 
quema plena y enfriamiento). Para la realización de este experimento, se usaron los siguientes equipos:

- Unidad de adquisición de datos que permite convertir la señal análoga enviada por los termopares y convertirla en señal digital de temperatura.

- Termopar tipo K, diseñada para soportar temperaturas de 0 a $1.500{ }^{\circ} \mathrm{C}$.

- Termopar tipo $\mathrm{K}$ revestida, que posee todas las características de la tipo $\mathrm{K}$ sencillo, pero esta tiene revestimiento cerámico, de tal forma que pueda usarse en el interior del horno.

- Computador, donde son almacenados los datos para un posterior procesamiento de la información.

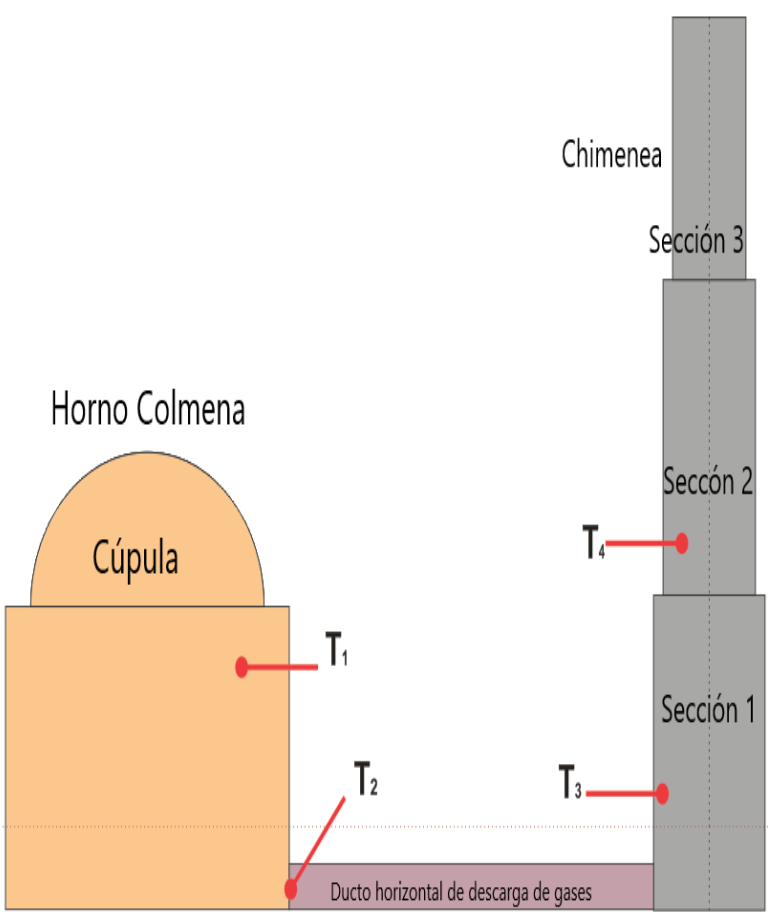

Figura 8. Puntos de medición

En la figura 9 se representa graficamente el lugar escogido para la instalacion del sistema recuperador de calor (IC), que aproveche el calor residual, en la etapa de quema plena, de los gases de combustión que salen de los hornos colmena. Para el diseño del IC, se considera lo siguiente:

- La disponibilidad de los materiales dentro del mercado.

- Los materiales a emplear deben ser de bajo costo y alta resistencia a la corrosión e incrustaciones, principalmente.
Tabla 2. Parámetros utilizados en el modelo matemático

\begin{tabular}{lr}
\hline \multicolumn{1}{c}{ Característica } & Valor \\
\hline Combustible utilizado & Carbón \\
Consumo de combustible [ton] & 21 \\
Número de productos & 24000 \\
Peso de cada producto húmedo [kg] & 5,35 \\
Peso de cada producto seco [kg] & 4,5 \\
Diámetro interno horno [m] & 11 \\
Diámetro externo horno [m] & 12,76 \\
Espesor del horno [m] & 0,76 \\
Altura total del horno [m] & 5,75 \\
Altura del horno antes de la cúpula [m] & 2,75 \\
Espesor de la cúpula [m] & 0,3 \\
Ancho de la puerta [m] & 1,5 \\
Alto de la puerta [m] & 2 \\
Espesor de la puerta [m] & 0,1 \\
Longitud ducto horizontal de descarga [m] & 6,7 \\
Altura ducto horizontal [m] & 0,8 \\
Ancho ducto horizontal [m] & 0,75 \\
Espesor del ducto horizontal [m] & 0,2 \\
Altura chimenea [m] & 17 \\
Temperatura ambiente [ $\left.{ }^{\circ} \mathrm{C}\right]$ & 26 \\
Velocidad del aire [km h ${ }^{-1}$ ] & 4 \\
Conductividad de la puerta [W/m K] & 0,658 \\
Conductividad del horno [W/m K] & 0,658 \\
Conductividad de la tierra [W/m K] & 1,5 \\
Conductividad de los productos [W/m K] & 0,94 \\
Conductividad de la chimenea [W/m K] & 0,658 \\
Rugosidad de los productos [mm] & 5 \\
Emisividad de la pared del horno & 0,91 \\
Emisividad de los productos & 0,94 \\
Emisividad de la pared de la chimenea & 0,91 \\
Emisividad del ducto horizontal & 0,85 \\
Entalpia del aire [kJ kg ${ }^{-1]}$ & 297,84 \\
\hline Fuent [4,15,14]
\end{tabular}

Fuente: $[4,15,14]$

\subsection{Sistema recuperador de calor}

- Utilizar los equipos existentes en la empresa.

- Optimizar el espacio disponible en la fábrica.

- El sistema de recuperación de calor solo se usará en la etapa de quema plena, ya que en la etapa de enfriamiento resulta más eficiente la recuperación implementada en la empresa, que consiste en extraer directamente el calor residual en los hornos y transportarlo a los secaderos.

- Se debe tener especial cuidado con el tiro de la chimenea. Si este se ve afectado, puede dañar la quema, $\mathrm{y}$, por lo tanto, dañará también los productos dentro del horno. 
- El aire precalentado se lleva a los secaderos por medio de los ductos utilizados para la recuperación de calor en la fase de enfriamiento.

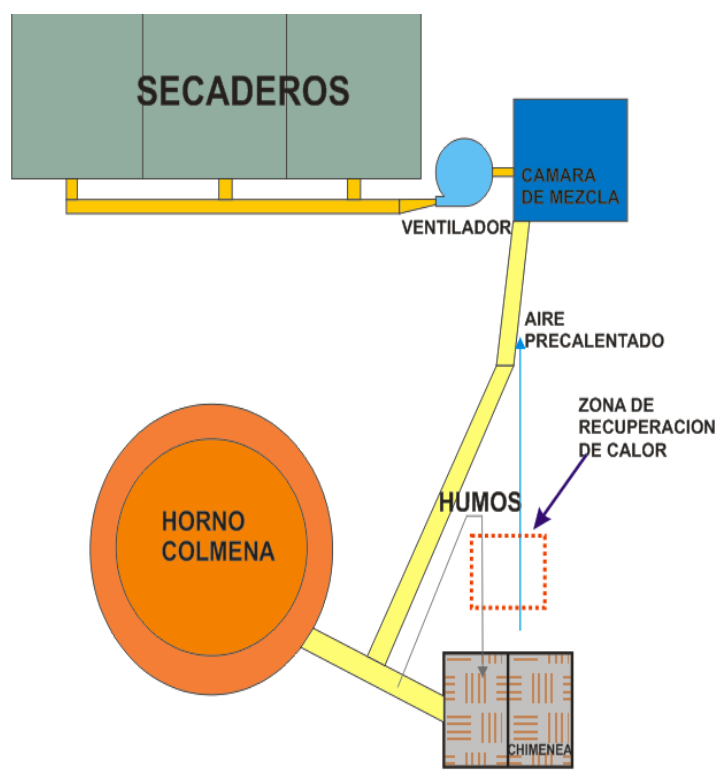

Figura 9. Esquema general de la planta y ubicación del IC

\subsubsection{Selección del sistema de recuperación}

En la industria existen diferentes sistemas recuperadores de calor, que permiten aprovechar esta energía residual. La teoría de recuperación de calor provee múltiples alternativas de solución para emplear en el ahorro de energía en la industria [3]. A partir de esto, se plantearon cinco posibles sistemas de recuperación: recuperación de calor usando aceite térmico, recuperación de calor usando un intercambiador de placas o compacto, recuperación de calor usando un regenerador Ljungstrom, recuperación de calor usando tubos de calor (heat pipe) y recuperación de calor desviando los humos de combustión directamente a los secaderos.

Se realizaó una revisión de la literatura sobre las alterativas presentadas anteriormente, esta revisión tuvo en cuenta aspectos relevantes, como la capacidad de intercambio de calor (eficiencia y tipo de fluido de intercambio de calor), el sistema de operación (tiempo de puesta en marcha, sistema de apagado, nivel de ruido, seguridad en la operación), la caída de presión, el mantenimiento (tiempo de reparo en caso de parada no programada, confiabilidad, disponibilidad de piezas para reparos y mantenimientos preventivos y correctivos), la resistencia (temperatura, corrosión y ensuciamiento), el cumplimiento de normas ambientales y los costos (adquisición y mantenimiento). Basados en esta información y en los objetivos de la empresa, se definieron los siguientes criterios a ser considerados en la matriz de decisión: eficiencia; costos iniciales, de operación y de mantenimiento; cumplimiento de la normativa ambiental y de seguridad y funcionamiento correcto en las condiciones de operación requeridas, para cada una de las alternativas. De esta forma, la información de la revisión literaria fue compartida con la empresa, para conocer su punto de vista, de modo que, como responsable del proyecto, pudiera decidir por medio de un sistema de ponderación la alternativa que más se ajustara a sus objetivos. El sistema de ponderación consiste en cuatro puntuaciones de la siguiente forma:

- 9 puntos, la alternativa cumple con el criterio de forma total $\rightarrow$ fuerte.

- 3 puntos, la alternativa cumple con el criterio de forma parcial $\rightarrow$ bueno.

- 1 punto, la alternativa cumple con el criterio de forma baja $\rightarrow$ medio.

- 0 puntos $\rightarrow$ la alternativa no cumple con el criterio $\rightarrow$ nulo

\subsubsection{Diseño del sistema IC}

El procedimiento de diseño para el IC fue adaptado de [3], y para un intercambiador de placas y aletas tiene los siguientes pasos:

1. Con las temperaturas de entrada y salida de cada fluido se calcula la temperatura media de los gases en el intercambiador y se determinan las propiedades termofísicas de cada fluido.

2. Se halla el flujo másico de aire, a partir del salto entálpico de los fluidos. El calor entregado por los gases es igual al calor que gana el aire. Al igualar las ecuaciones (21) y (22), se obtiene el flujo másico del aire $\dot{m}_{\text {aire }}$.

$$
\begin{gathered}
q_{g a s}=m_{g a s} C_{p g a s}\left(T_{g, i}-T_{g, s}\right) \\
q_{\text {aire }}=m_{\text {aire }} C_{p a}\left(T_{a, s}-T_{a, i}\right)
\end{gathered}
$$

Donde

- $\dot{m}_{g a s}$ es el flujo másico de los gases $\left[\mathrm{m} \mathrm{s}^{-1}\right]$.

- $C_{\text {pgas }}$ y $C_{p a}$ son los calores específicos de los gases y del aire respectivamente $\left[\mathrm{kJ} \mathrm{kg}^{-1}\right]$.

- $T_{g, i}$ y $T_{a, i}$ son las temperaturas de entrada de los gases y el aire respectivamente $\left[{ }^{\circ} \mathrm{C}\right]$.

- $T_{g, s}$ y $T_{a, s}$ son las temperaturas de salida de los gases y del aire respectivamente $\left[{ }^{\circ} \mathrm{C}\right]$.

3. Conocidos los dos flujos se sabe que el $\mathrm{C}_{\min }$ es el de los gases de combustión; por lo tanto, $\mathrm{C}_{\max }$ es el del 
aire. Se calcula la relación de capacidades caloríficas $\mathrm{R}$, ecuación (23)

$$
R=\frac{C_{\min }}{C_{\max }}=\frac{m_{\text {gas }} C_{\text {pgas }}}{m_{\text {aire }} C_{p a}}
$$

4. El delta de temperatura máximo para el caso de un intercambiador de flujo cruzado es

$$
\Delta T_{\max }=\left(T_{g, i}-T_{a, i}\right)
$$

5. Conociendo el delta de temperatura máximo, es posible determinar el calor máximo posible a intercambiar.

$$
q_{\max }=m_{g a s} C_{p g a s} \Delta T_{\max }
$$

6. Se calcula la eficiencia teórica del intercambiador.

$$
\varepsilon=\frac{q_{\text {real }}}{q_{\max }}=\frac{q_{\text {aire }}}{q_{\max }}=\frac{q_{\text {gas }}}{q_{\max }}
$$

7. La eficiencia real de un intercambiador de placas y aletas es calculada utilizando la ecuación (27). Para calcular la eficiencia real, es necesario suponer las características geométricas superficiales para un intercambiador de placas y aletas, y así calcular los grupos adimensionales con los que se realizan el análisis de transferencia de calor, las pérdidas de presión y la eficiencia del intercambiador [16].

$$
\varepsilon=1-e^{\left[\left(\frac{1}{R}\right) N U T^{0.22}\left(e^{\left[-R(-N U T)^{0.78}\right]-1}\right)\right]}
$$

8. $\quad \boldsymbol{U} \boldsymbol{T}$ es el número de unidades de transferencia de calor. Se calcula utilizando la ecuación (28) [16].

$$
N U T=\frac{U A}{C_{\min }}
$$

9. Se calcula el coeficiente global de transferencia de calor y área (UA), con base en las impurezas depositadas en la superficie por los humos de combustión del carbón $\mathrm{R}_{\mathrm{f}} \mathrm{y}$ los efectos de aletas (superficie extendida), utilizando la ecuación (29).

$$
\frac{1}{U A}=\frac{1}{\eta_{o} h_{c} A_{c}}+R_{f}+\frac{d w}{A_{w} k_{w, i c}}+\frac{1}{\eta_{o} h_{h} A_{h}}
$$

Donde

- $\eta_{o, c}$ es la eficiencia de la superficie aleteada, lado aire.

- $\eta_{o, h}$ es la eficiencia de la superficie aleteada, lado gases.
- $A_{c} y A_{h}$ son las áreas totales, lado aire y gases, respectivamente $\left[\mathrm{m}^{2}\right]$.

- $A_{w}$ es el área de la pared $\left[\mathrm{m}^{2}\right]$.

- $h_{c} y h_{h}$ son los coeficientes de transferencia de calor convectivo, lado gases y aire, respectivamente $\left[\mathrm{W} / \mathrm{m}^{2}\right.$ $\mathrm{K}]$.

- $d_{w}$ es el espesor de la placa [m].

- $k_{w}$ es la conductividad térmica de la placa [W/m K].

10. El coeficiente de transferencia de calor por convección se calcula utilizado las ecuaciones (7) a la (10), donde $k$ es la conductividad térmica de los gases o del aire $[\mathrm{W} / \mathrm{m} \mathrm{K}$ y y la longitud característica es el diámetro hidráulico del intercambiador $D_{h}=$ $4 r_{h}$, según la geometría supuesta.

11. Así, se calculan los grupos adimensionales que se necesitan para determinar las características; el Nusselt se evalúa usando la fórmula de Gnielinski [14]:

$$
N_{u}=\frac{\frac{f}{8}\left(R_{e}-1000\right) P_{r}}{\left[1+12.7\left(\sqrt{\frac{f}{8}\left(P_{r}^{2 / 3}-1\right)}\right)\right]}
$$

Donde

- $R_{e}$ es el número de Reynolds.

- $P_{r}$ es el número de Prandtl, que depende del tipo de fluido y temperatura, y se calcula como en la ecuación (10).

- $f$ es el factor de fricción de Fanning, que depende de la rugosidad del material $e, D_{h}$ y del $R_{e}$.

$$
f=\frac{0.25}{\left(\log \left(\frac{1}{3.7 \frac{D_{h}}{e}}+\frac{5.74}{R_{e}^{0.9}}\right)\right)^{2}}
$$

12. Con los valores de los parámetros de los pasos 8 al 12 , se calcula la eficiencia real del intercambiador (paso 7), y se compara el valor de la eficiencia teórica (paso 6). Si la diferencia entre estas es significativa, se asume un área de transferencia diferente (aumentando el número de pasos para cada fluido) en el paso 7 , y se realiza nuevamente el proceso iterativo del paso 7 al 11 hasta que las eficiencias sean similares. 
13. Con el área real se calcula la pérdida de presión para cada fluido, a través del intercambiador, usando la ecuación (32) [10].

$$
\Delta P=\frac{G^{2}}{2 g_{c} \rho_{i}}\left[\left(1-\sigma^{2}+K_{c}\right)+2\left(\frac{\rho_{i}}{\rho_{o}}-1\right)+f \frac{L}{r_{h}} \rho_{i}\left(\frac{1}{\rho}\right)_{m}-\left(1-\sigma^{2}-K_{e}\right) \frac{\rho_{i}}{\rho_{o}}\right]
$$

Donde

- $G=\frac{\dot{m}}{A_{o}}$ es la velocidad de masa, y depende del flujo másico del fluido y del área transversal de la sección del intercambiador $\left[\mathrm{m} \mathrm{s}^{-1}\right]$.

- $\quad r_{h}=\frac{A_{o}}{P}$ es el radio hidráulico que depende del área y del perímetro [m].

- $\quad \rho_{i} y \rho_{o}$ es la densidad del fluido a la temperatura de entrada y salida del intercambiador, respectivamente $\left[\mathrm{kg} \mathrm{m}^{-3}\right]$.

- El volumen específico medio $\left[\mathrm{m}^{3} \mathrm{~kg}\right]$ se puede expresar de la siguiente manera:

$$
\left(\frac{1}{\rho}\right)_{m}=\frac{1}{2}\left(\frac{1}{\rho_{i}}+\frac{1}{\rho_{o}}\right)
$$

- $\quad L$ es la longitud del ducto [m].

- $K_{c} y K_{e}$ son los factores de pérdida de presión en la entrada y salida, respectivamente.

- $\sigma$ es la relación entre el área transversal del flujo a la entrada y el área transversal en el núcleo del ducto.

\subsection{Cálculo de costos}

Se determina el precio de construcción, adquisición y operación de los elementos que componen el sistema. Para esto se utilizó la información suministrada por la página Matches [18]. El precio de construcción de un intercambiador de calor se basa en la función exponencial, a partir la información disponible, la ecuación (34) describe la función exponencial para el cálculo de costos para el tipo de intercambiador analizado, donde $\mathrm{A}$ es el área de transferencia de calor total del equipo $\left[\mathrm{m}^{2}\right]$.

$$
C I C=\left(6 \times 10^{6}\right) A^{0.5494}
$$

En la tabla 3 se presentan los parámetros utilizados para

\begin{tabular}{|c|c|}
\hline \multicolumn{2}{|l|}{ Parámetros } \\
\hline Factor de ensuciamiento $\boldsymbol{R}_{\boldsymbol{f}}$ & 0,881 \\
\hline Rugosidad de los ductos del horno $\boldsymbol{e}_{\boldsymbol{d}}[\mathrm{mm}]$ & 9 \\
\hline Rugosidad de los ductos del intercambiador & 0,045 \\
\hline $\boldsymbol{e}_{\text {int }}[\mathrm{mm}]$ & \\
\hline $\begin{array}{l}\text { Conductividad térmica de la placa } \boldsymbol{k}_{\boldsymbol{w}}[\mathrm{W} / \mathrm{m} \\
\mathrm{K}]\end{array}$ & 50 \\
\hline factor de pérdida de presión en la entrada $\boldsymbol{K}_{c}$ & 0,5 \\
\hline factor de pérdida de presión en la salida $\boldsymbol{K}_{\boldsymbol{e}}$ & 0,35 \\
\hline Precio del kilovatio hora de energía eléctrica & 240 \\
\hline para el sector industrial $\boldsymbol{C}_{\boldsymbol{k} W \boldsymbol{h}}\left[\frac{C O P \$}{V W h}\right]$ & \\
\hline índice de precios al consumidor $\mathbf{F}$ & 0,04 \\
\hline Variador de velocidad CA [ millones] & \\
\hline $\begin{array}{l}\text { Tiempo de trabajo del equipo } \mathbf{t}[\text { horas por } \\
\text { año] }\end{array}$ & 2.160 \\
\hline
\end{tabular}
el diseño del intercambiador de calor.
Tabla 3. Parámetros usados en el diseño del IC

Fuente: $[4,14,16]$

Para impulsar el aire a través del intercambiador de calor se usa el ventilador disponible en la empresa, que tiene un motor con potencia de $30 \mathrm{hp}$, el diámetro de su turbina es de 45,25 pulgadas, la velocidad del motor es $1.765 \mathrm{rpm}$ y el flujo volumétrico es 2.2954,53 CFM. El costo de bombeo es un factor muy importante para tener en cuenta en los costos de operación, y se calcula utilizando la ecuación (35).

$$
C B=\left[\left(\frac{V \Delta P}{e}\right) \times t \times\left(\frac{C_{k W h} F}{1000}\right)\right]
$$

Donde

- $\Delta P$ es la carga que debe ser impulsada $[\mathrm{Pa}]$.

- $\dot{V}$ es el caudal $\left[\mathrm{m}^{3} \mathrm{~s}^{-1}\right]$.

- $e$ es la eficiencia de bombeo del equipo.

- $t$ es el tiempo de trabajo del equipo, trabaja 45 horas semanales [h].

- $C_{k W h}$ es el precio del kilovatio hora de energía eléctrica para el sector industrial $\left[\frac{C O P \$}{k W h}\right]$.

- F es el índice de precios al consumidor.

\section{Resultados y discusiones}

En primer lugar, se presentan los resultados obtenidos usando el modelo matemático para calcular la temperatura de los gases dentro del horno, cuando se varía la forma en que se hace el suministro de carbón al horno. En la figura 10 se presentan los datos de temperatura obtenidos cuando se ingresa el flujo másico de carbón constante, es decir, carbón total gastado en la 


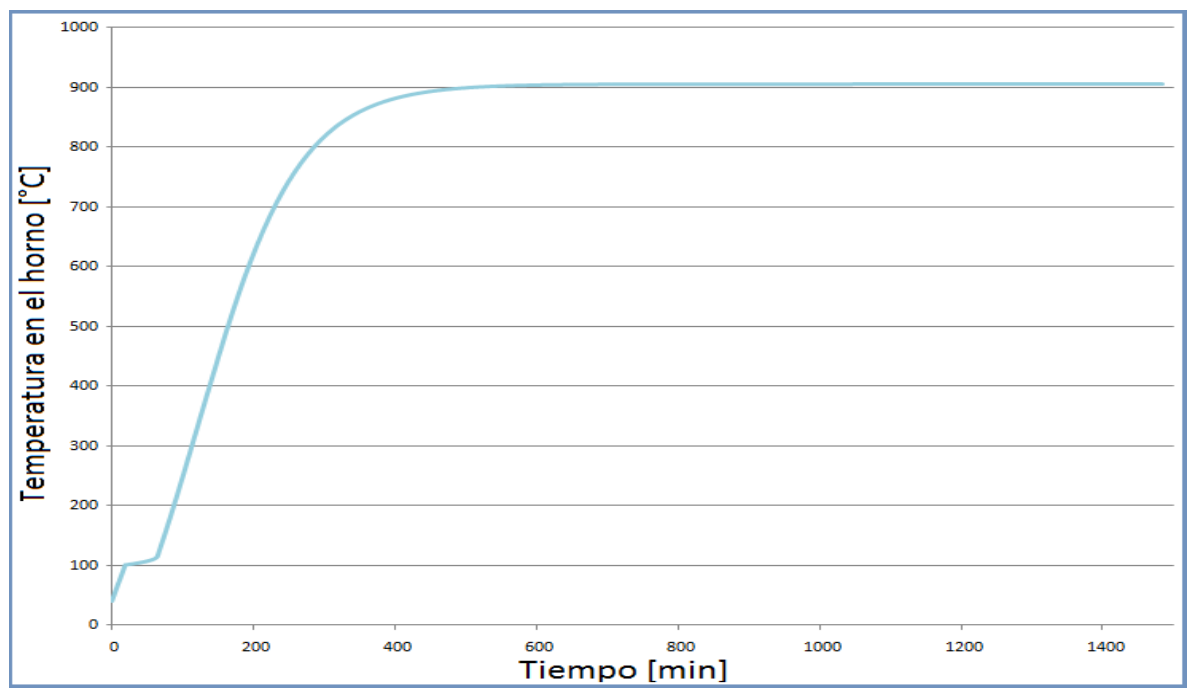

Figura 10. Temperatura simulada en el horno con flujo másico de carbón de alimentación constante

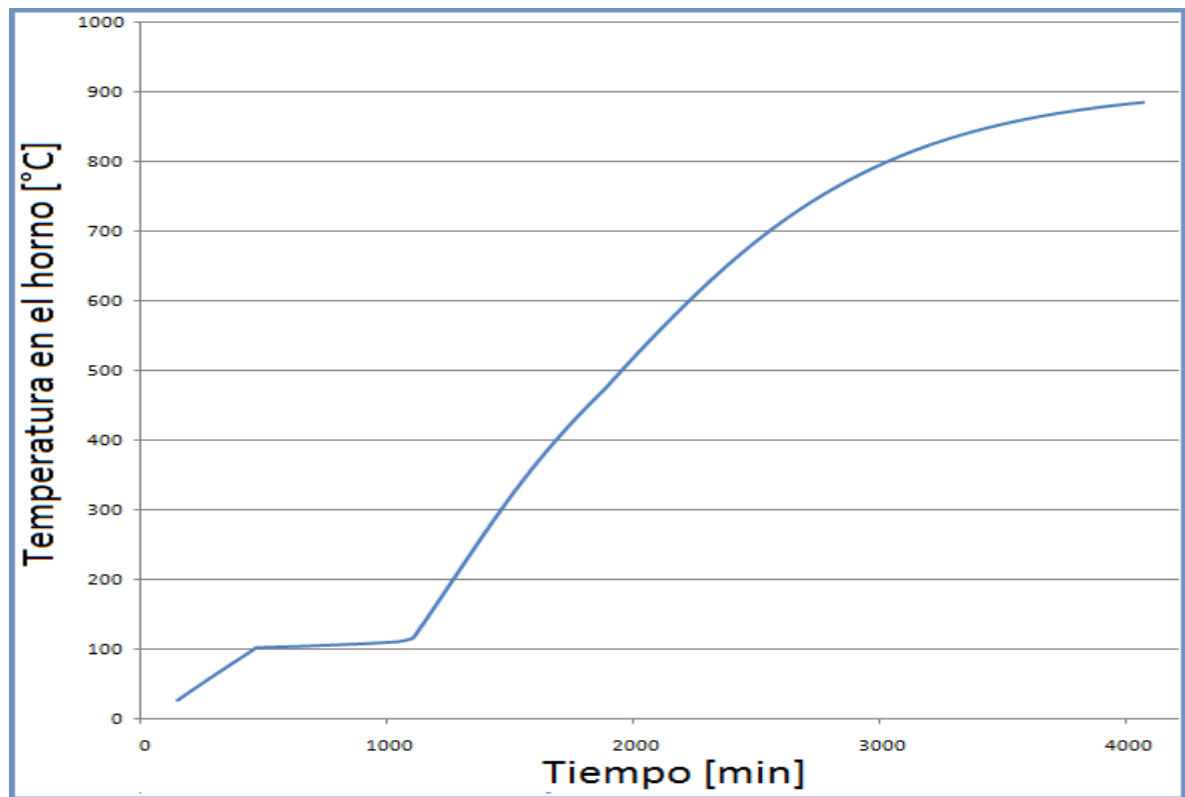

Figura 11. Temperatura simulada en el horno con flujo másico de carbón de alimentación variable

quema dividido en el tiempo total de quema. Se observa que la temperatura de los gases se eleva hasta $905^{\circ} \mathrm{C}$ en tan solo $500 \mathrm{~min}$. Esto quiere decir que el horno alcanzaría temperaturas muy elevadas en corto tiempo, y esto afectaría la correcta cocción de los productos.

En la figura 11 se presentan los datos de temperaturas que alcanzan los humos dentro del horno, según el ingreso del flujo másico del carbón en el modelo matemático como una función lineal del tiempo, derivada del carbón total entregado.

Se observa que la temperatura de los gases tiene un inicio más plano y luego se incrementa rápidamente desde los
$1.200 \mathrm{~min}$ a los $4.000 \mathrm{~min}$. La pendiente en la curva de la temperatura rebasa los datos de operación del horno dentro de este rango de tiempo, pero al final de la quema se aproxima a los valores de operación real. El aumento de temperatura después de los 1.200 min afecta la etapa de caldeo, ya que a los $500 \mathrm{~min}$ la temperatura de los gases dentro del horno es aproximadamente $150{ }^{\circ} \mathrm{C}$ más que la necesaria.

Por ende, se debe modificar la forma en que se ingresa el valor de esta variable al código desarrollado.

Finalmente, se ingresa un flujo másico del carbón en el modelo matemático como una función del tiempo, 
derivada de los estudios experimentales realizados. Los resultados de las temperaturas se muestran como una línea en la figura 12. En esta se puede observar que las temperaturas simuladas se aproximan a los datos experimentales, con lo cual se considera que el modelo matemático es capaz de reproducir los fenómenos físicos presentes en el horno. Además, son comparados los resultados simulados con respecto a los datos experimentales medidos en los cuatro puntos de medición descritos anteriormente. Los símbolos azules muestran la temperatura $\mathrm{T} 1$, los rojos la $\mathrm{T} 2$, los verdes la T3 y los morados la T4.

Como se observa en la figura 12, durante la etapa de caldeo (hasta los $1.500 \mathrm{~min}$ ), el incremento en la temperatura no es sustancial, debido a que el gasto energético necesario para evaporar el agua presente en los productos es alto. Además, la cantidad de carbón suministrado en esta etapa inicial es casi la mitad del flujo utilizado en la etapa de quema plena.

La etapa de quema plena comprende desde los $1.500 \mathrm{~min}$ hasta los $3.900 \mathrm{~min}$. En este rango de tiempo se puede hacer el proceso de recuperación de calor, ya que las temperaturas alcanzadas por los gases en el ducto y la chimenea superan los $200{ }^{\circ} \mathrm{C}$. Además, muestra que la temperatura modelada de los gases en el horno está en medio de los datos experimentales T1 y T2. Esto se debe a que los gases en el punto de medición T1 aún no han cedido calor a los productos y paredes, por eso son más altos. Mientras que en T2, ducto de salida del horno, los gases ya han intercambiado calor con las paredes y los productos, y han perdido buena parte de su energía.

\subsection{Selección del tipo de intercambiador}

En la tabla 3 se presentan los valores asignados para cada uno de los aspectos de cada sistema. Se selecciona el que mayor puntaje obtenga. Así, se puede apreciar que la alternativa de solución que mejor cumple con las condiciones de operación y con las necesidades de la empresa es el sistema que usa un intercambiador compacto, ya que ha obtenido la mayor puntuación. Se diseña un intercambiador de calor de placas aleteadas en flujo cruzado.

En la figura 14 se muestra un esquema de esta alternativa. Consiste en la instalación de un intercambiador de calor de placas o compacto, a través del cual pasan los gases de combustión y el aire ambiente. La recuperación empieza cuando se desvían los gases de combustión por cierto número de pasos que contienen placas corrugadas, la superficie constituida por estas placas transportará el calor residual presente en los gases y reducirá su temperatura. Al mismo tiempo, se hará pasar aire ambiente en flujo cruzado por los canales intermedios, y absorberá el calor cedido por los gases.

Tabla 3. Evaluación para seleccionar la mejor alternativa de recuperación.

\begin{tabular}{|c|c|c|c|c|c|}
\hline $\begin{array}{l}\text { Sistema } \\
\text { Criterio }\end{array}$ & $\begin{array}{l}\text { Aceite } \\
\text { térmico }\end{array}$ & Compacto & Ljustrom & $\begin{array}{c}\text { Tubos } \\
\text { de } \\
\text { calor }\end{array}$ & Desvío \\
\hline Eficiencia & 3 & 3 & 9 & 3 & 1 \\
\hline Costo inicial & 1 & 3 & 0 & 3 & 9 \\
\hline $\begin{array}{l}\text { Costo equipos } \\
\text { de impulsión }\end{array}$ & 1 & 3 & 1 & 3 & 3 \\
\hline $\begin{array}{l}\text { Costo } \\
\text { manteni- } \\
\text { miento }\end{array}$ & 1 & 1 & 1 & 1 & 3 \\
\hline $\begin{array}{l}\text { Normas } \\
\text { ambientales }\end{array}$ & 3 & 9 & 9 & 3 & 1 \\
\hline $\begin{array}{l}\text { Normas } \\
\text { seguridad }\end{array}$ & 3 & 9 & 3 & 9 & 1 \\
\hline $\begin{array}{l}\text { Costo de } \\
\text { operación }\end{array}$ & 1 & 3 & 1 & 3 & 9 \\
\hline $\begin{array}{l}\text { Funciona- } \\
\text { miento }\end{array}$ & 9 & 9 & 9 & 1 & 3 \\
\hline TOTAL & 22 & 40 & 33 & 26 & 30 \\
\hline
\end{tabular}

Fuente: Autores

El intercambiador de calor usado para aprovechar la energía residual trabaja con los siguientes parámetros de diseño:

- Temperatura de entrada de los gases $T_{g, i}$ mínima 250 ${ }^{\circ} \mathrm{C}$ y máxima $550{ }^{\circ} \mathrm{C}$.

- Por normas ambientales, la temperatura de los gases a la salida del recuperador $T_{g, s}$ debe ser menor de $180{ }^{\circ} \mathrm{C}$ [19].

- El flujo másico de los gases es $2.642\left[\mathrm{~kg} \mathrm{~s}^{-1}\right]$

- Las propiedades de los gases dependen de la temperatura. El calor específico a $300{ }^{\circ} \mathrm{C}$ es $1.121,5$ [J/kgK].

- En el proceso de secado, el aire debe estar en un rango de temperatura entre $60{ }^{\circ} \mathrm{C}$ y $90^{\circ} \mathrm{C}$, temperaturas que se deben alcanzar con el recuperador.

- Temperatura del aire ambiente es $24^{\circ} \mathrm{C}$, y esta será la temperatura del aire al entrar en el recuperador.

- El flujo másico del aire es 7,28 [kg s$\left.{ }^{-1}\right]$.

- El calor residual máximo y mínimo disponible en los gases de combustión $q$ es:

$$
\begin{aligned}
& q=m_{g a s} C_{p g}\left(T_{g, i}-T_{g, s}\right) \\
& q_{\min }=207.4[k W] \\
& q_{\max }=984.1[k W]
\end{aligned}
$$




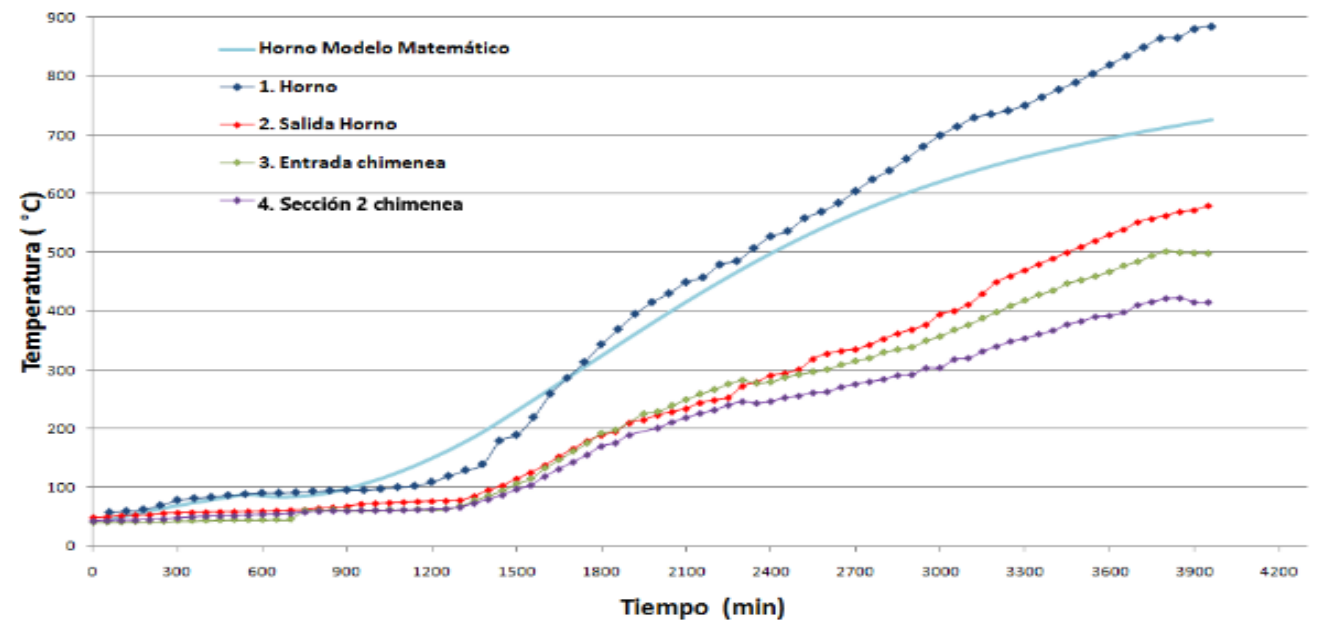

Figura 12. Temperatura de los gases de combustión en el horno. Medidos (símbolos). Modelados (línea)

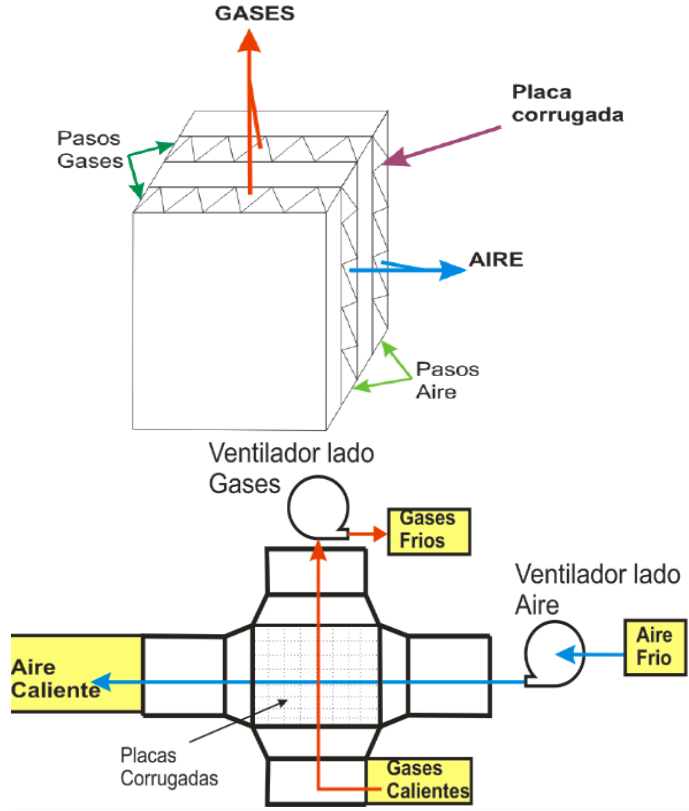

Figura 13. Configuración sistema recuperador con placas o compacto.

El sistema de recuperación estará integrado por una serie de placas corrugadas orientadas en el sentido de los flujos. Cada flujo tendrá un número definido de pasos óptimos, para garantizar la recuperación más eficiente. En cuanto al lado gases, el punto crítico de operación se encuentra en la salida del intercambiador. Debido a que la temperatura de salida es $180{ }^{\circ} \mathrm{C}$, se seleccionó un ventilador de la marca Soler \& Palau, puesto que ofrece equipos que operan con estas temperaturas. El modelo seleccionado fue BIL 450, y tiene las siguientes características:
- Caudal 2,31 $\left[\mathrm{m}^{3} \mathrm{~s}^{-1}\right]$

- Presión 3,7 [kPa]

- Diámetro de la turbina 455 [mm]

- Velocidad del motor 2885 [rpm]

- Potencia del motor 9,94 [hp]

Para el análisis de los puntos de operación, se desarrolló una herramienta de diseño y aplicación que permite conocer las condiciones geométricas del equipo, el caudal necesario del aire y las pérdidas de presión. Esto a partir de las temperaturas de entrada y salida del aire y del gas. La interfaz gráfica de la aplicación se muestra en la figura 14. En el cuadro rojo (1) se ingresan los valores de las temperaturas de los gases y el aire. En los cuadros verdes (2) se obtiene el análisis de costos. Así mismo, se encuentran las gráficas de área frente a costos de bombeo, área frente a costo de construcción y la gráfica que contiene la suma de los costos, de donde se escoge la opción que se ajusta a las necesidades.

En el cuadro morado (3) se genera una gráfica donde se compara la altura de la aleta (canal) y el área de transferencia de calor. Y, por último, en el cuadro naranja (4) se observan los valores resultantes de la geometría, la presión, el flujo y el costo de la mejor opción para este punto de operación. En la tabla 4 se presentan las características principales del sistema recuperador de calor, y en la tabla 5, los costos del sistema.

Como se ve en la figura 15 el sistema recuperador tiene como equipo principal un intercambiador compacto o de placas aleteadas, y cuenta con un sistema de impulsión integrado por una serie de ductos y dos ventiladores para el transporte de los fluidos. 


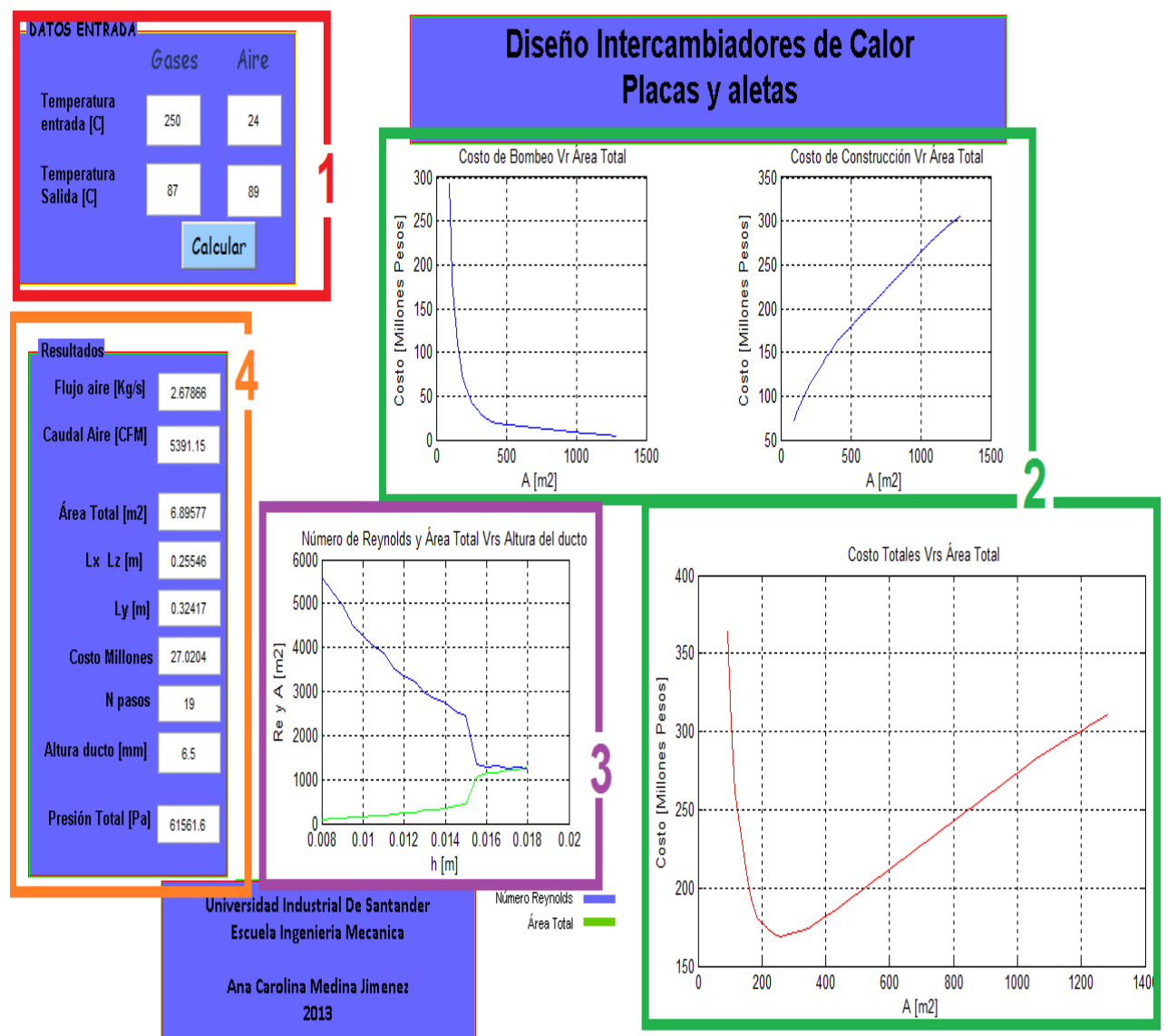

Figura 14. Interfaz gráfica herramienta de diseño

\section{Conclusiones}

Se desarrolló un modelo matemático para calcularla:

- Se desarrolló un modelo matemático para calcular la temperatura de los gases dentro del horno colmena. En el modelo se tuvieron en cuenta los fenómenos de transferencia de calor y masa entre gases y productos, pérdidas energéticas por las paredes del horno y se consideró la variación de las propiedades físicas de los gases con la temperatura mediante un análisis termodinámico de la combustión. El modelo matemático se validó con los datos experimentales.

- Para la alimentación de carbón se usó una aproximación matemática del proceso real, pues este es escalonado y aumenta de acuerdo con el criterio del operario, y el carbón no se suministra a todas las bocas simultáneamente.
Tabla 4. Características del intercambiador de calor compacto

\begin{tabular}{|c|c|}
\hline Características & \\
\hline Área superficial del intercambiador $\left[\mathrm{m}^{2}\right]$ & 52,46 \\
\hline Alto $[\mathrm{m}]$ & 0,669 \\
\hline Ancho $[\mathrm{m}]$ & 0,563 \\
\hline Largo $[\mathrm{m}]$ & 0,563 \\
\hline Número de pasos lado aire & 29 \\
\hline Número de pasos lado gases & 28 \\
\hline Número de aletas entre placas & 57 \\
\hline $\begin{array}{l}\text { Distancia entre placas y altura de los canales } \\
\text { [mm] }\end{array}$ & 9,5 \\
\hline Espesor de las aletas $[\mathrm{mm}]$ & 0,31 \\
\hline Espesor de las placas [mm] & 1,9 \\
\hline Temperatura de los gases a la entrada $\left[{ }^{\circ} \mathrm{C}\right]$ & 500 \\
\hline Temperatura de los gases a la salida $\left[{ }^{\circ} \mathrm{C}\right]$ & 180 \\
\hline Temperatura del aire a la entrada $\left[{ }^{\circ} \mathrm{C}\right]$ & 24 \\
\hline Temperatura del aire a la salida $\left[{ }^{\circ} \mathrm{C}\right]$ & 110 \\
\hline Caudal de aire [CFM] & 22.571 \\
\hline Caída de presión en el intercambiador $[\mathrm{kPa}]$ & 40.5 \\
\hline Energía recuperada por el IC [kW] & 549,9 \\
\hline
\end{tabular}

Fuente: $[4,14,16]$ 

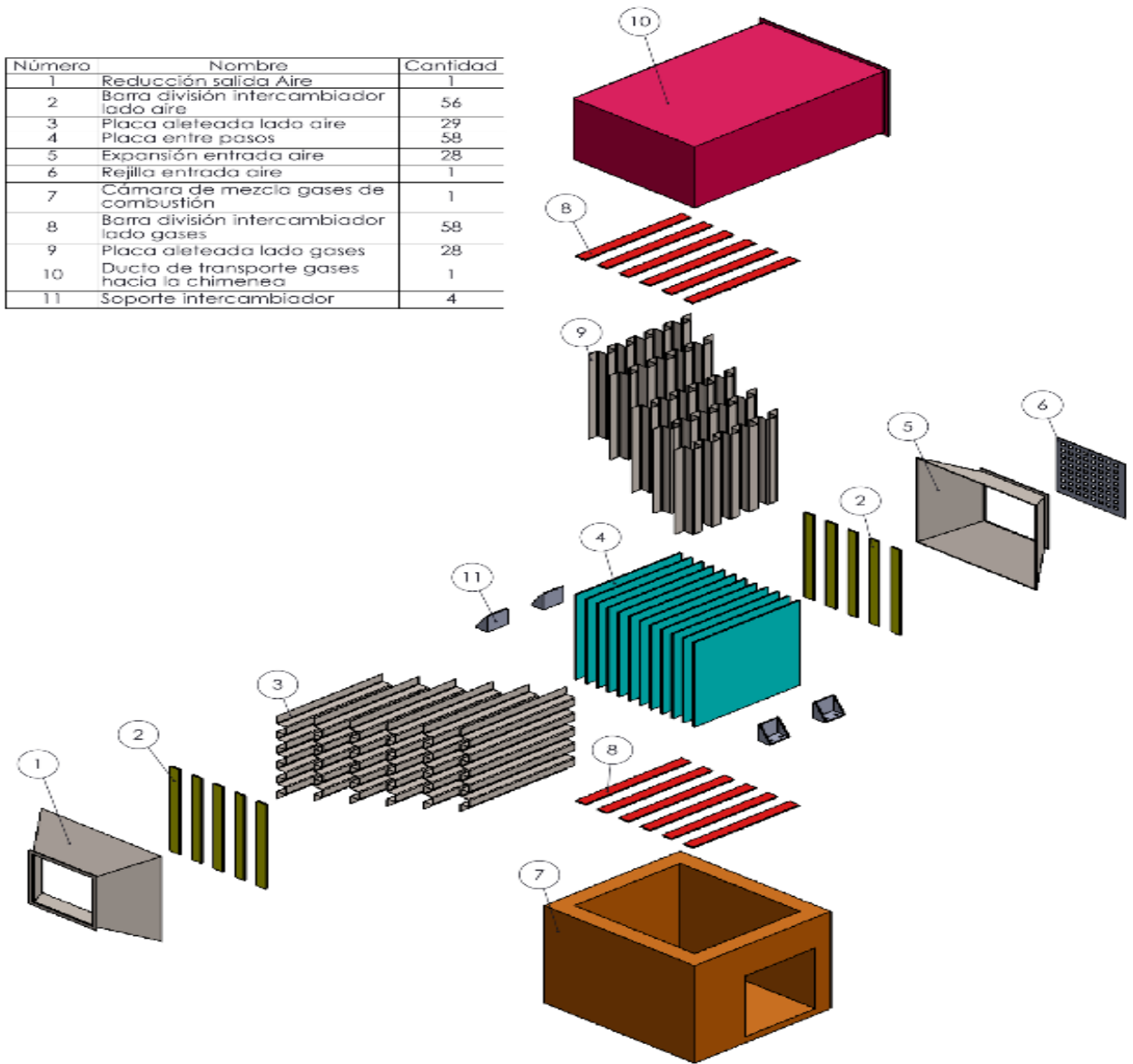

Figura 15. Interfaz gráfica herramienta de diseño

Tabla 5. Costos del sistema

\begin{tabular}{lll}
\hline \multicolumn{1}{c}{ Ítem } & \multicolumn{1}{c}{ Referencia } & Precio [COP] \\
\hline $\begin{array}{l}\text { Intercambiador } \\
\text { de calor }\end{array}$ & $\begin{array}{l}\text { Acero } \\
\text { inoxidable }\end{array}$ & 52 ' 848.900 \\
$\begin{array}{l}\text { AISI 304L } \\
\text { Auctos de }\end{array}$ & $\begin{array}{l}\text { Acero } \\
\text { galvanizado }\end{array}$ & 2 2'100,000 \\
$\begin{array}{l}\text { Ventilador } \\
\text { centrífugo tubular }\end{array}$ & BIL 450 & $133^{\prime} 000.000$ \\
\hline
\end{tabular}

- Se realizó una preselección de cinco alternativas para el sistema de recuperación de calor, con base en las temperaturas medidas en el estudio experimental realizado en la empresa. Se definieron los criterios básicos a tener en cuenta en el diseño del sistema recuperador. De acuerdo con estos criterios, se seleccionó un tipo de recuperador de calor de flujo cruzado de placas aleteadas. Posteriormente, se realizó el diseño detallado de este y se obtuvieron las características mostradas en la Tabla 4. Características del intercambiador de calor compacto. Con este sistema

de recuperación la empresa tendrá un ahorro energético de $549 \mathrm{~kW}$

- Se recomienda a la empresa mantener un mejor control en la forma en que se entrega el carbón al horno, pues es una variable crítica en el proceso de cocción, como se demostró con el análisis paramétrico realizado con el modelo desarrollado con la entrega de carbón constante (figura 10), que eleva rápidamente la temperatura de los gases dentro del horno, y variable (figura 11).

\section{Agradecimientos}

Los autores agradecen el apoyo financiero brindado por la Universidad Industrial de Santander por medio del proyecto 1358 .

\section{Referencias}

[1] M. A. Ramírez-Argáez, S. L. Huacúz, and G. Trápaga, "Mathematical Modeling of Pottery Production in Different Industrial Furnaces," J. Mater. Eng. 
Perform., vol. 17, no. 5, pp. 633-643, 2008. doi: 10.1007/s11665-008-9217-5

[2] J. Sáncheza, S. M. Rozo-Rincón, J. Gelves-Díaz, and J. Bautista-Ruiz, "Caracterización de cerámicos inmersos en soluciones del sistema sio2 - tio2 - zro2 sintetizadas por el método sol-gel," Rev. UIS Ing., vol. 16, no. 2, pp. 51-60, 2017. doi: 10.18273/revuin.v16n2-2017005

[3] R. K. Shah and D. P. Sekulic, Fundamentals of heat exchanger design. United States of America: John Wiley \& Sons, 2003.

[4] A. C. Medina Jimenez, "Diseño de un sistema para la recuperación del calor residual en los gases de combustión de los hornos colmena en la empresa Ladrillos y acabados S.A.S.," trabajo de fin de grado, Universidad Industrial de Santander, 2013.

[5] P. Mullinger and B. Jenkins, Industrial and Process Furnaces: principles, design and operation. Oxford: Elsevier, 2008.

[6] M. Moran, H. Shapiro, D. Boettner, and M. Bailey, Fundamentals of Engineering Thermodynamics. United States of America: John Wiley \& Sons, 2011.

[7] C. E. Baukal, Heat Transfer in Industrial Combustion. United States of America: CRC Press, 2000.

[8] T. L. Bergman, A. S. Lavine, F. P. Incropera, and D. P. Dewitt, Fundamentals of Heat and Mass Transfer. United States of America: John Wiley \& Sons, 2011.

[9] K. Asano, Mass Transfer: from fndamentals to modern industral applications. Wiley-VCH, 2006.

[10] R. L. Mott, Mecánica de Fluidos. México: Pearson Educación, 2006.

[11] CRANE, Flujos de fluidos en válvulas, accesorios y tuberías. McGraw-Hill, 1987.

[12] A. Mezquita, E. Monfort, E. Vaquer, S. Ferrer, M. A. Arnal, J. Toledo, and M. A. Cuesta, "Optimización energética en la fabricación de baldosas cerámicas mediante el uso de aceite térmico," Boletín la Soc. Española Cerámica y Vidr., vol. 51, no. 4, pp. 183-190, 2014. doi: 10.3989/cyv.152014

[13] P. Díez, "Centrales Térmicas," 2000. [Online]. Available: http://es.pfernandezdiez.es/libro/?id=15. [Accessed: 02-Feb-2013].
[14] A. F. Mills, Transferencia de Calor. Los Angeles, 1991.

[15] S. Lucia, M. Parra, and L. A. Sanchez, "Desarrollo de una propuesta de optimización energética de los hornos colmena de la empresa ladrillos y acabados S.A.S," trabajo de fin de grado, Universidad Industrial de Santander, 2011.

[16] W. M. Rohsenow, J. P. Hartnett, and Y. I. Cho, Handbook of heat transfer. United States of America: McGraw-Hill, 1998.

[17] S. Enterprises, "Scilab," 2012. [Online]. Available: https://www.scilab.org. [Accessed: 01-Feb-2012].

[18] Matches, "Matches' Process Equipment Cost Estimates,” 2014.

[19] Por la cual se establecen las normas y estándares de emisión admisibles de contaminantes a la atmósfera por fuentes fijas y se dictan otras disposiciones. Resolución 909 de junio de 2008, Bogotá. 